\title{
Magnet Experiment to Measuring Space Propulsion Heim-Lorentz Force
}

\author{
Walter Dröscher ${ }^{1}$, Jochem Hauser ${ }^{1,2}$
}

This paper describes in a non-mathematical way, by using a sequence of pictures, the physics of a novel space propulsion technique and its experimental realization, based on a unified field theory in a quantized, 8-dimensional space, developed by the late German physicist Burkhard Heim, termed Heim Quantum Theory (HQT or $H T$ ). Following a strict geometrization principle introduced by the first author, HQT predicts six fundamental interactions, requiring two additional gravitational like interactions, represented by gravitophotons (attractive and repulsive, fifth interaction), and the quintessence or vacuum particle (repulsive, sixth interaction), enabling a completely different type of propulsion, denoted gravitophoton field propulsion. The gravitophoton force, would accelerate a material body without the need of propellant. Gravitophoton particles are generated in pairs from the vacuum itself by the effect of vacuum polarization (virtual electrons), under the presence of a very strong magnetic field (photons). Attractive gravitophotons interact with matter, and thus can become real particles, exacting a force on a material body. In particular, the experimental setup is described to measure the so called gravitational Heim-Lorentz force, which is a result of gravitophoton pair production. Experimental conditions are discussed with emphasis to magnet design to obtaining the high magnetic field strengths to generate an appreciable Heim-Lorentz force.

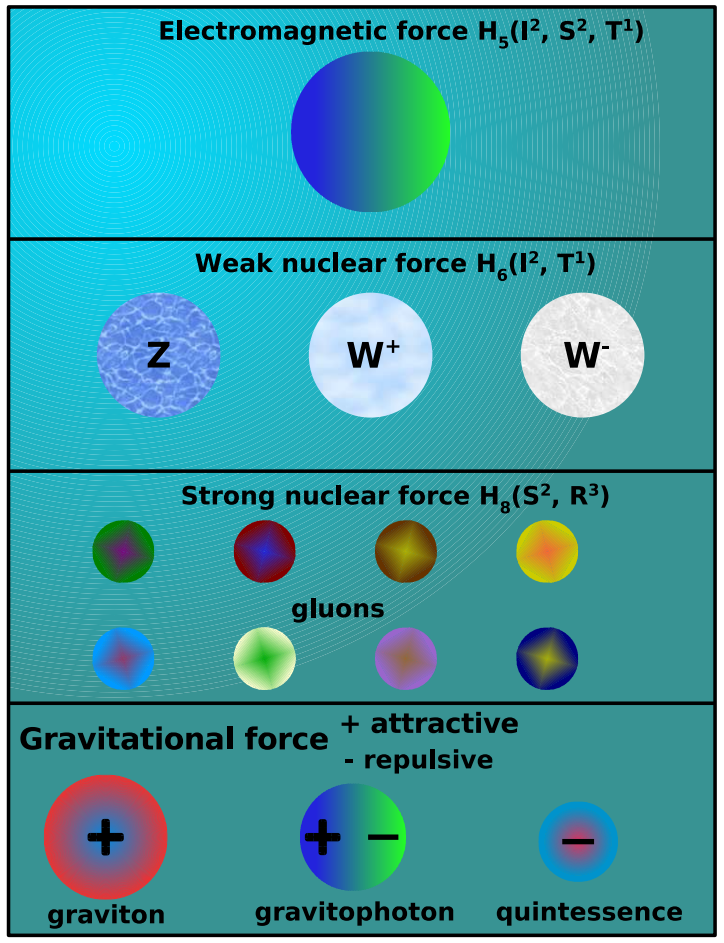

1 Institut für Grenzgebiete der Wissenschaft (IGW), Leopold - Franzens Universität Innsbruck, Innsbruck, Austria 2 Faculty Karl-Scharfenberg, University of Applied Sciences, Salzgitter, Germany

1 Senior scientist, 2 Senior member AIAA, member SSE, Prof., www.hpcc-space.com or www.uibk.ac.at/c/cb/cb26/

(C) 2005 Institut für Grenzgebiete der Wissenschaft, Leopold - Franzens Universität Innsbruck, Austria 


\section{Field Propulsion Principles according to Heim Theory $^{2}$}

Heim went beyond general relativity and asked the question: if the effects of the gravitational field can be described by a connection (Christoffel symbols) in spacetime that describes the relative orientation between local coordinate frames in spacetime, can all other forces of nature such as electromagnetism, the weak force, and the strong force be associated with respective connections or an equivalent metric tensor.

Clearly, this must lead to a higher dimensional space, since in $G R$ spacetime gives rise to only one interaction, which is gravity. Furthermore, the assumption of locality for all physical interactions is of greatest importance and determines the general structure of a unified theory as well as the number of fundamental physical interactions.

Following this geometrization principle (Sec. 2) leads to a poly-metric that describes all fundamental interactions, but also predicts two additional gravitational like interactions represented by massless particles named gravitophoton (both attractive and repulsive), and a particle termed quintessence or vacuum particle (repulsive) that is identified with dark energy. Due to gravitophoton pair production, the total energy extracted from the vacuum is zero. In addition, the theory predicts the conversion of photons into gravitophotons and, under special conditions (very strong magnetic fields), the conversion into quintessence particles. Since the coupling constants for these two additional gravitational interactions can be calculated in Heim's theory, quantitative predictions of the gravitophoton interaction are possible. The equation describing this force is termed the Heim-Lorentz equation, because of its structural similarity with the magnetic Lorentz force (mathematical details are given in $[13,14])$. However, the Heim-Lorentz force is a gravitational force, generated by pair production of gravitophotons (attractive, graviton like) and repulsive from the vacuum. No energy is extracted from the vacuum, but the cross section of the attractive (or negative, with regard to energy) gravitophoton for interaction with matter is much larger than for the repulsive (or positive) gravitophoton, so

2 The picture on the front cover depicts the six fundamental interactions resulting from $H Q T$. There should exist two additional gravitational like forces that can be both attractive and repulsive. The gravitophoton particles are generated from photons, and thus $H Q T$ predicts an interaction between electromagnetism and gravitation. The letter $\mathrm{H}$ with an index (indices 6 and 8 in the picture are not correct, see Table 3) stands for hermetry form, a concept described in Sec. 2.1.

3 For nomenclature and glossary see www.hpcc-space.com under Publications: HeimTheoryGlossary.pdf that a net force on material objects is exerted once gravitophoton pair production begins. In Secs. 3 and 4 a discussion of the experimental realization of the Heim-Lorentz force is presented.

The theory also predicts an interaction of repulsive gravitophotons with gravitons, for instance the gravitophotons of the spacecraft, leading to a production of quintessence particles, and consequently reducing its gravitational potential $\Phi$. This would require either a reduction of the mass of the spacecraft or a reduction of the magnitude of the gravitational constant $G$. For a reduced mass, conservation of momentum would require a velocity $c^{\prime}>c$ in $\mathbb{R}^{4}$. Both, Heim and recent loop gravity theory do predict a quantized minimal surface area which is $\tau=8 \pi \sqrt{3} \hbar G c^{-3}$. The factor

$8 \pi \sqrt{3}$ cannot be determined directly from loop quantum gravity, but was chosen to fit the Bekenstein-Hawking formula for the entropy of a black hole [7, 9]. Heim performed a phenomenological derivation and obtained the factor $3 / 4 \pi$. Any physical phenomenon requiring a gravitational constant $G^{\prime}<G$ or a speed of light $c^{\prime}>c$ in $\mathbb{R}^{4}$ therefore has to be ruled out, violating the fact that $\mathrm{T}$ is the minimum surface. On the other hand, because of positive gravitophoton action, $\Phi$ is actually reduced. In order to resolve this contradiction, it is postulated that the object has to leave our spacetime and enters into a parallel space (or parallel universe or multiverse), denoted as $\mathbb{R}^{4}$ (n) with $n \in \mathbb{N}$. It seems to be possible that a spacecraft, under certain conditions, stated below by Eq. 12, will be able to enter into such a parallel space. Covariant physical laws hold in parallel space $\mathbb{R}^{4}(n)$ with respect to $\mathbb{R}^{4}$, but the gravitational constant, the mass of the spacecraft, and the speed of light are changed according to $G(n)=G / n, M$ $(n)=n M$, and $c(n)=n c$ where $n$ is larger than 1 . In such a space, superluminal speeds would be possible in principle. The interesting fact is that an object can transit into parallel space at a relatively low speed from our own spacetime $\mathbb{R}^{4}$, so no excessive energy input is needed.

\section{Heim Quantum Field Theory and the Physics of Elementary Particles}

Einstein, in 1950 [9], emphasized the principle of geometrization of all physical interactions. The importance of GR is that there exists no background coordinate system. Therefore, conventional quantum field theories that are relying on such a background space will not be suc- 
cessful in constructing a quantum theory of gravity. In how far string theory $[1,3], S T$, that uses a background metric will be able to recover background independence is something that seems undecided at present. On the contrary, according to Einstein, one should start with $G R$ and incorporate the quantum principle. This is the approach followed by Heim and also by Rovelli, Smolin and Ashtekar et al. [4, 5, 7, 10, 12]. In addition, spacetime in these theories is discrete. It is known that the general theory of relativity $(G R)$ in a 4-dimensional spacetime delivers one possible physical interaction, namely gravitation. Since Nature shows us that there exist additional interactions (EM, weak, strong), and because both $G R$ and the quantum principle are experimentally verified, it seems logical to extend the geometrical principle to a discrete, higher-dimensional space. Furthermore, the spontaneous order that has been observed in the universe is opposite to the laws of thermodynamics, predicting the increase of disorder or greater entropy [6]. Everywhere highly evolved structures can be seen, which is an enigma for the science of today. Consequently, the theory utilizes an entelechial dimension, $\mathrm{x}^{5}$, an aeonic dimension, $\mathrm{x}^{6}$ forming subspace $S^{2}$ (see glossary), and coordinates $x^{7}, x^{8}$ describing information forming subspace $\mathrm{I}^{2}$, i.e., quantum mechanics, resulting in an 8-dimensional discrete space in which a smallest elemental surface, the so-called metron, exists. $\mathrm{H}^{8}$ comprises real fields, the hermetry forms, producing real physical effects. One of these hermetry forms, $\mathrm{H}_{12}$, is responsible for gravity, but there are 11 other hermetry forms plus 3 degenerated hermetry forms, part of them listed in Table 3 . The physics in $H Q T$ is therefore determined by the poly-metric of the hermetry forms. This kind of poly-metric is currently not included in quantum field theory, loop quantum gravity, or string theory.

\subsection{Hermetry Forms and Physical Interac- tions}

In this paper we present the physical ideas of the geometrization concept underlying Heim theory in 8D space using a series of pictures, see Figs. 1- 6. The mathematical derivation for hermetry forms was given in $[13,14]$. As described in $[13,14]$ there is a general coordinate transformation $x^{m}\left(\xi^{\alpha}\left(\eta^{i}\right)\right)$ from $\mathbb{R}^{4} \rightarrow \mathrm{H}^{8} \rightarrow \mathbb{R}^{4}$ resulting in the metric tensor

$$
g_{i k}=\frac{\partial x^{m}}{\partial \xi^{\alpha}} \frac{\partial \xi^{\alpha}}{\partial \eta^{i}} \frac{\partial x^{m}}{\partial \xi^{\beta}} \frac{\partial \xi^{\beta}}{\partial \eta^{k}}
$$

where indices $\alpha, \beta=1, \ldots, 8$ and $i, m, k=1, \ldots, 4$. The Einstein summation convention is used, that is, indices occurring twice are summed over.

$$
g_{i k}=: \sum_{\mu, v=1}^{8} g_{i k}^{(\mu \nu)}
$$

$$
g_{i k}^{(\mu \nu)}=\frac{\partial x^{m}}{\partial \xi^{(\mu)}} \frac{\partial \xi^{(\mu)}}{\partial \eta^{i}} \frac{\partial x^{m}}{\partial \xi^{(v)}} \frac{\partial \xi^{(v)}}{\partial \eta^{k}}
$$

Twelve hermetry forms can be generated having direct physical meaning, by constructing specific combinations from the four subspaces. The following denotation for the metric describing hermetry form $H_{\ell}$ with $\ell=1, \ldots, 12$ is used:

$$
g_{i k}\left(H_{\ell}\right)=: \sum_{\mu, v \in H_{\ell}} g_{i k}^{(\mu v)}
$$

where summation indices are obtained from the definition of the hermetry forms. The expressions $g_{i k}\left(H_{\ell}\right)$ are interpreted as different physical interaction potentials caused by hermetry form $\mathrm{H}_{\ell}$, extending the interpretation of metric employed in $G R$ to the poly-metric of $\mathrm{H}^{8}$. It should be noted that any valid hermetry form either must contain space $\mathrm{S}^{2}$ or $\mathrm{I}^{2}$.

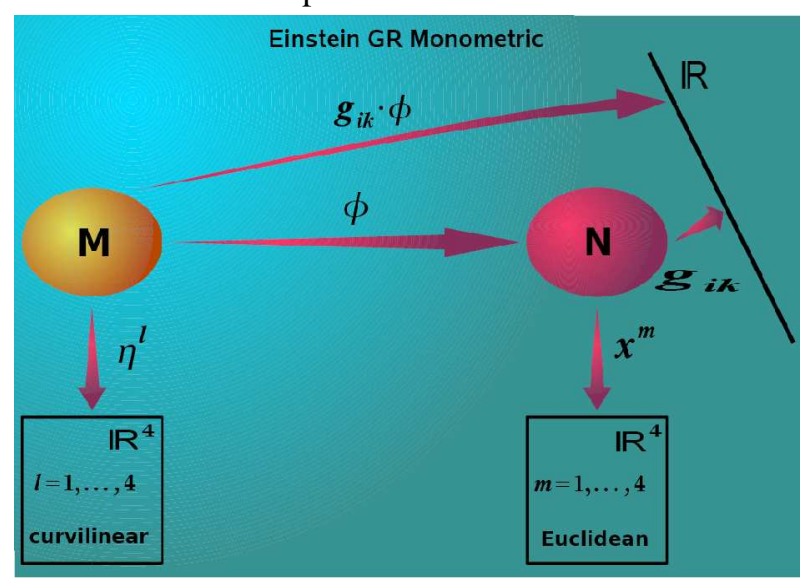

Figure 1: In GR the metric tensor is computed using a mapping from manifold $M$ (curvilinear coordinates $\eta^{\prime}$ ) in spacetime $\mathbb{R}^{4}$ to manifold $N$ in spacetime $\mathbb{R}^{4}$ (Euclidean coordinates $\left.x^{m}\right)$. Calculating the components of the metric tensor as well as lengths, areas, and volumes from the metric tensor a mapping to the set of real numbers is needed [17]. This kind of mapping delivers one type of metric tensor that is responsible for gravity, appearing on the LHS of the Einstein field equations.

Each individual hermetry form is equivalent to a physical potential or a messenger particle. It should be noted that spaces $\mathrm{S}^{2} \times \mathrm{I}^{2}$ describe gravitophotons and $\mathrm{S}^{2} \times \mathrm{I}^{2} \times \mathrm{T}^{1}$ are responsible for photons. 


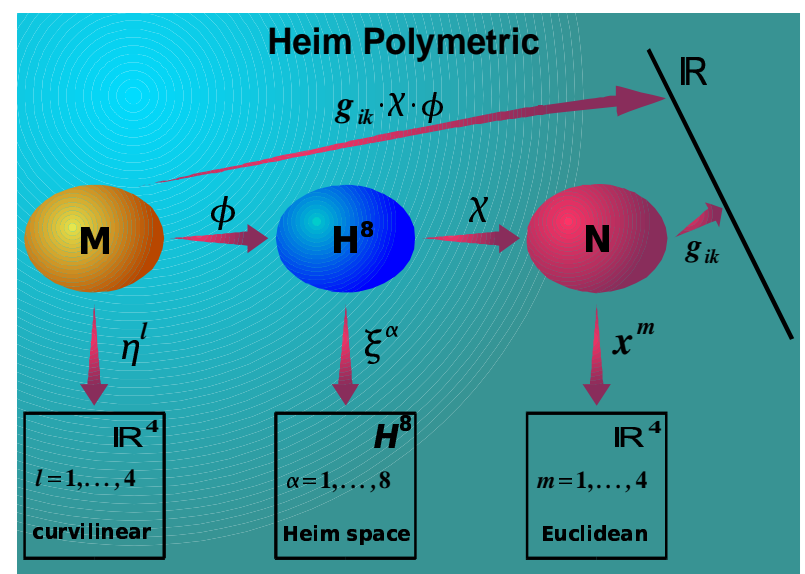

Figure 2: Einstein's goal was the unification of all physical interactions based on his principle of geometrization, i.e., having a metric that is responsible for the interaction. This principle is termed Einstein's geometrization principle of physics (EGP). To this end, Heim and Dröscher introduced the concept of an internal space, denoted as Heim space $\mathrm{H}^{8}$, having 8 dimensions. Although $\mathrm{H}^{8}$ is not a physical space, the signature of the additional coordinates being timelike (negative), these invisible internal coordinates govern events in spacetime. Therefore, a mapping from manifold $M$ (curvilinear coordinates $\eta^{\prime}$ ) in spacetime $\mathbb{R}^{4}$ to internal space $\mathrm{H}^{8}$ and back to manifold $N$ in spacetime $\mathbb{R}^{4}$ (Euclidean coordinates $\left.x^{m}\right)$ must be used to properly describe the physics. This is a major deviation from GR and leads to a poly-metric. For the construction of the poly-metric see Eqs. (2) and (4).

\subsection{Transformation Equations and Coupling Constants}

There are two equations describing the conversion of photons into pairs of gravitophotons, Eqs. $(5,6)$. The first equation describes the production of $N^{2}$ gravitophoton particles from photons.

$$
\begin{aligned}
& w_{p h}(r)-w_{p h}=N w_{g p} \\
& w_{p h}(r)-w_{p h}=A w_{p h} .
\end{aligned}
$$

This equation is obtained from Heim's theory in 8D space in combination with considerations from number theory, and predicts the conversion of photons into gravitophoton particles. The second equation is taken from Landau's, radiation correction.

Conversion amplitude: The physical meaning of Eqs. $(5,6)$ is that an electromagnetic potential (photon) containing probability amplitude $A w_{p h}$ can be converted into

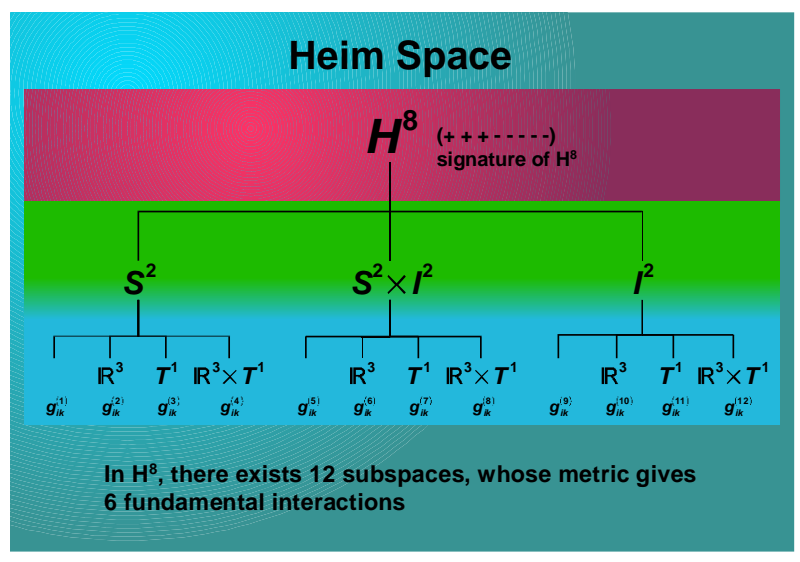

Figure 3: In Heim space there are four additional internal coordinates with timelike (negative) signature, giving rise to two additional subspaces $\mathrm{S}^{2}$ and $\mathrm{I}^{2}$. Hence, $\mathrm{H}^{8}$ comprises four subspaces, namely $\mathbb{R}^{3}, \mathrm{~T}^{1}, \mathrm{~S}^{2}$, and $\mathrm{I}^{2}$. The picture shows the kind of metric-subspace that can be constructed, where each element is denoted as a hermetry form. Each hermetry form has a direct physical meaning, see Table 3. In order to construct a hermetry form, either internal space $\mathrm{S}^{2}$ or $\mathrm{I}^{2}$ must be present. In addition, there are two degenerated hermetry forms that describe partial forms of the photon and the quintessence potential. They allow the conversion of photons into gravitophotons as well as of gravitophotons and gravitons into quintessence particles.

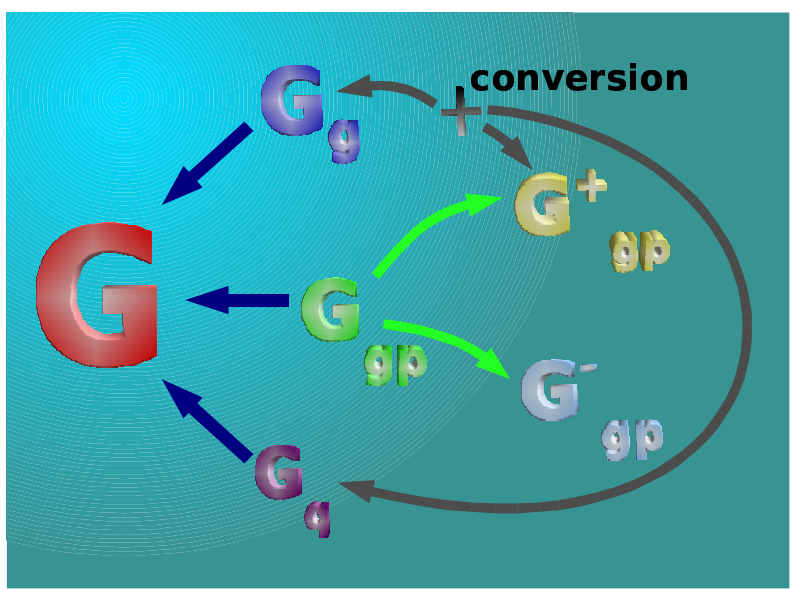

Figure 4: There should be three gravitational particles, namely the graviton (attractive), the gravitophoton (attractive and repulsive), and the quintessence or vacuum particle (repulsive), represented by hermetry forms $H_{5}, H_{11}$, and $H_{12}$, see Table 3.

a gravitophoton potential (pair of gravitophotons) with associated probability amplitude $N w_{g p}$. From Eqs. $(5,6)$ the following relation needs to be satisfied for gravitophoton production, requiring the existence of a shielding potential that has to be provided by experiment. The function $A(\mathrm{r})$ can be calculated from Landau's radiation correction with numerical values for $A$ ranging from $10^{-3}$ to $10^{-4}$. From Eqs. (5) one obtains

$$
N w_{g p}=A w_{p h} .
$$




\section{Heim-Lorentz Force for Space Pro- pulsion}

The Heim-Lorentz force derived in [13] is the basis for the field propulsion mechanism. In this section a description of the physical processes for the generation of the Heim-Lorentz force is presented along with the experimental setup. It turns out that several conditions need to be satisfied. In particular, very high magnetic field strengths are required. This issue is addressed in Sec. 4.

\subsection{Heim-Lorentz Force}

Eqs. (7) and (8) are the so called Heim-Lorentz force which is a gravitational force.

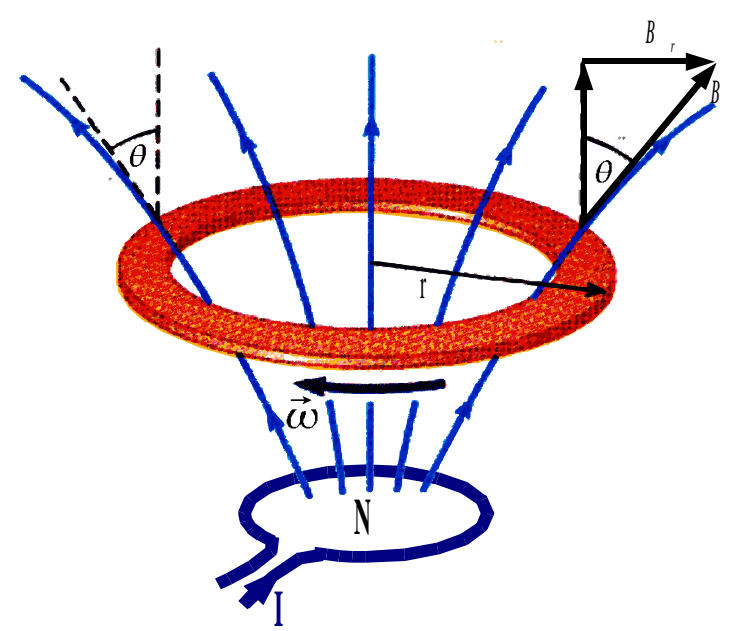

Figure 5: This picture shows the experimental setup to measuring the Heim-Lorentz force. The current loop (blue) provides an inhomogeneous magnetic field at the location of the rotating torus (red). The radial field component causes a gradient in the z-direction (vertical). The experimental setup also would serve as the field propulsion system, if appropriately dimensioned. For very high magnetic fields over $30 \mathrm{~T}$, the current loop or solenoid must be mechanically reinforced because of the Lorentz force acting on the moving electrons in the solenoid, forcing them toward the center of the loop.

$$
F_{g p}=-\Lambda_{p} e \mu_{0} v^{T} \times H
$$

where $\Lambda_{p}$ indicates that only proton and neutron absorption processes were considered in $[13,14]$. is determined as

$$
\frac{32}{3}\left(\frac{N w_{g p e}}{w_{p h}}\right)^{2}\left(N w_{g p a}\right)^{4}\left(\frac{\hbar}{m_{p} c}\right)^{2} \frac{d}{d_{0}^{3}} Z .
$$

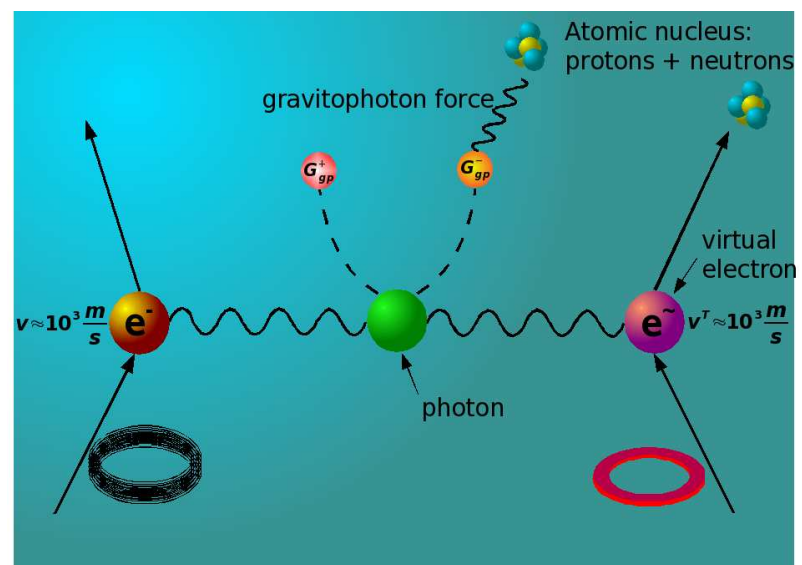

Figure 6: This picture shows the physical phenomenon of gravitophoton pair production from the vacuum, using some kind of Feynman diagram. Gravitophoton production from photons is the cause of the Heim-Lorentz force. The brown sphere on the LHS corresponds to the electrons moving in the current loop, Fig. 5. The sphere on the RHS corresponds to the rotating ring above the current loop, having a circumferential speed of some $10^{3} \mathrm{~m} / \mathrm{s}$. In the rotating torus virtual electrons are produced by the vacuum, partially shielding the proton charge of the nuclei. At a distance smaller than the Compton wavelength of the electron away from the nucleus, the proton charge increases, since it is less shielded. According to Eq. (6) a value of A larger than 0 is needed for gravitophoton production. As was shown in [12], however, a smaller value of $A$ is needed to start converting photons into gravitophotons to make the photon metric vanish, termed $\tilde{A}=v_{k} v_{k}^{T} / c^{2} \approx 10^{-11}$ where $v$ is the velocity of the electrons in the current loop and $v^{T}$ is the circumferential speed of the torus. From the vanishing photon metric, the metric of the gravitophoton pairs is generated, replacing the value of $A$ by the LHS of Eq. (6) and inserting it into the equation for the gravitophoton metric. This value is then increased to the value of $A$ in Eq.(6). Experimentally this is achieved by the current loop (magnetic coil) that generates the magnetic vector and the tensor potential at the location of the virtual electron in the rotating torus, producing a high enough product $v v^{T}$ see Eq. 32 in [13]. The coupling constants of the two gravitophoton particles are different, and only the negative (attractive) gravitophotons are absorbed by protons and neutrons, while absorption by electrons can be neglected. This is plausible since the negative (attractive) gravitophoton contains the metric of the graviton, while the positive repulsive gravitophoton contains the metric of the quintessence particle that does only interact extremely weakly with matter. Through the interaction of the attractive gravitophoton with matter it becomes a real particle and thus a measurable force is generated (see upper part of the picture).

$\Lambda_{p}$ (dimensionless) is a highly nonlinear function of the probability amplitude of the gravitophoton particle.

It is important to note that Eq. (7) only describes the acceleration stage of gravitophoton field propulsion.

There is a distance $r_{N}$ at which the shielded electric potential produced by the nucleus and the magnetic potential cancel, given by Eq. 30 in [13]

$$
r_{N}=\frac{Z e}{Q} R \frac{c}{v_{i}} \frac{c}{v_{i}^{T}}
$$


Due to the shielding of the proton charges in the nucleus by virtual electrons coming out of the spacetime field (or vacuum), for distances $r<r_{\mathrm{N}}$, the atomic number is a function of distance from the nucleus that is, $Z=Z(r)$. At distances $r_{\mathrm{N}}$ smaller than the Compton wavelength of the electron, the bare charge of the proton gradually becomes visible, as expressed by $A(r)$. From Eq. (6) it is obvious that a value of $A$ larger than 0 is needed for gravitophoton production and from Eq. (9) it can be seen that a small value of $r_{\mathrm{N}}$ is obtained by high velocities of the electrons in the current loop as well as a high speed of the rotating torus. A more detailed analysis for $r_{\mathrm{N}}$ accounts for the fact that $Q$ is not a point charge. The value for $r_{\mathrm{N}}$ turns out to be

$$
r_{N}=\frac{Z e c^{2}}{4 \operatorname{In} v_{i}^{T}}
$$

where $I$ is the current and $n$ is the number of turns in the solenoid (not to be confused with $n$ in Eq. (12)). A simple calculation for a rotating torus having a mass of $1,000 \mathrm{~kg}$ of hydrogen and a spacecraft mass of $10^{5} \mathrm{~kg}$, shows that a value of $N w_{\text {gpe }}=4.4 \times 10^{-5}$ is needed, where a magnetic induction of roughly $20 \mathrm{~T}$ is necessary for $I$ $n=4 \times 10^{7}$, and $\mathrm{d}=0.5 \mathrm{~m}, \mathrm{D}=6 \mathrm{~m}$, see Table 1 . The velocity of the torus is $700 \mathrm{~m} / \mathrm{s}$. The number of turns $n$ was assumed as $6.6 \times 10^{4}$. These values should be compared to Table 1. It should be noted that a torus of $6 \mathrm{~m}$ requires a fairly large experimental setup. Alternatively a smaller torus diameter requires a higher magnetic induction. The kinetic energy provided to the torus is $2.45 \times 10^{8}$ Joule which is substantial. However, it is a small amount compared to a spacecraft having a mass of $10^{5} \mathrm{~kg}$, flying at a speed of $1 \%$ of the speed of light, which carries an energy content of $4.5 \times 10^{17} \mathrm{~J}$. Even if the spacecraft can be provided with a $100 \mathrm{MW}$ nuclear reactor, it would take some 143 years to produce this amount of energy.

In the end, a detailed power and mass analysis has to be carried out to build the optimal field propulsion device.

It is not the value of the magnetic induction in the current loop, but it is the strength of the magnetic field $\mathbf{H}$ that is of importance. In other words, an iron core in a magnetic coil will not increase the production of graviton photon pairs from the vacuum. Hence, the value $\mu_{0} \mathbf{H}$ is listed in Table 1 .

\begin{tabular}{|c|c|c|c|c|c|}
\hline $\begin{array}{c}\mathbf{d} \\
{[\mathrm{m}]}\end{array}$ & $\begin{array}{c}\mathrm{D} \\
{[\mathrm{m}]}\end{array}$ & $\begin{array}{c}\boldsymbol{I n} \\
{[\mathrm{An}]}\end{array}$ & $\boldsymbol{N} \boldsymbol{w}_{\text {gpe }}$ & $\begin{array}{c}\boldsymbol{\mu}_{0} \boldsymbol{H} \\
(\boldsymbol{T})\end{array}$ & $\Delta$ \\
\hline 0.2 & 2 & $6.6 \times 10^{6}$ & $\begin{array}{r}1.4 \times 10^{-} \\
7\end{array}$ & 13 & $7 \times 10^{-16}$ \\
\hline 0.3 & 3 & $1.3 \times 10^{7}$ & $\begin{array}{r}7.4 \times 10^{-} \\
6\end{array}$ & 18 & $2 \times 10^{-5}$ \\
\hline 0.4 & 4 & $2.7 \times 10^{7}$ & $2 \times 10^{-5}$ & 27 & $\begin{array}{r}2.1 \times 10^{-} \\
2\end{array}$ \\
\hline 0.5 & 5 & $4 \times 10^{7}$ & $3.9 \times 10^{-5}$ & 33 & 0.72 \\
\hline 0.6 & 6 & $1.5 \times 10^{7}$ & $4.8 \times 10^{-5}$ & 38 & 3 \\
\hline
\end{tabular}

Table 1: From the Heim-Lorentz force the following values are obtained. A mass of only $100 \mathrm{~kg}$ of the torus is assumed, filled with $5 \mathrm{~kg}$ of hydrogen. The current density is 600 $\mathrm{A} / \mathrm{mm}^{2}$. The value $\Delta$ is the relative change with respect to earth acceleration $g=9.81 \mathrm{~m} / \mathrm{s}^{2}$ that can be achieved at the corresponding magnetic field strength. The value $\mu_{0} \mathbf{H}$ is the magnetic induction generated by the superconductor at the location of the rotating torus, $D$ is the major diameter of the torus, while $d$ is the minor diameter. In stands for the product of current and the number of turns of the magnetic coil. The velocity of the torus was assumed to be $700 \mathrm{~m} / \mathrm{s}$. Total wire length would be some $10^{6} \mathrm{~m}$. Assuming a reduction in voltage of $1 \mu \mathrm{V} / \mathrm{cm}$ for a superconductor, a thermal power of some $8 \mathrm{~kW}$ has to be managed. In general, a factor of 500 needs to be applied at $4.2 \mathrm{~K}$ to calculate the cooling power that amounts to some $4 M W$.

\subsection{Transition into Parallel Space}

Under the assumption that the gravitational potential of the spacecraft can be reduced by the production of quintessence particles as discussed in Sec.1., a transition into parallel space is postulated to avoid a potential conflict with relativity theory.

A parallel space $\mathbb{R}^{4}(n)$, in which covariant physical laws with respect to $\mathbb{R}^{4}$ exist, is characterized by the scaling transformation

$$
\begin{aligned}
& x_{i}(n)=\frac{1}{n^{2}} x(1), i=1,2,3 ; t(n)=\frac{1}{n^{3}} t(1) \\
& v(n)=n v(1) ; c(n)=n c(1) \\
& G(n)=\frac{1}{n} G ; \hbar(n)=\hbar ; n \in \mathbb{N} .
\end{aligned}
$$

The fact that $n$ must be an integer stems from the requirement in $H Q T$ and $L Q T$ for a smallest length scale. Hence only discrete and no continuous transformations are possible. The Lorentz transformation is invariant with regard to the transformations of Eqs. (11) ${ }^{4}$. In other words, physical laws should be covariant under discrete (quantized) spacetime dilatations (contractions). There are two important questions to be addressed, namely how the value $n$ can be influenced

4 It is straightforward to show that Einstein's field equations as well as the Friedmann equations are also invariant under dilatations. 
by experimental parameters, and how the back-transformation from $\mathbb{R}^{4}(n) \rightarrow \mathbb{R}^{4}$ is working. The result of the back-transformation must not depend on the choice of the origin of the coordinate system in $\mathbb{R}^{4}$. As a result of the combined mapping from $\mathbb{R}^{4} \rightarrow \mathbb{R}^{4}(n) \rightarrow \mathbb{R}^{4}$, the spacecraft has moved a distance $n v \Delta t$ when re-entering $\mathbb{R}^{4}$. The value $\Delta t$ denotes the time difference between leaving and reentering $\mathbb{R}^{4}$, as measured by an observer in $\mathbb{R}^{4}$. This mapping for the transformation of distance, time, and velocity differences cannot be the identity matrix. That is, the back transformation from $\mathbb{R}^{4}(n) \rightarrow$ $\mathbb{R}^{4}$ is not the inverse of the mapping from $\mathbb{R}^{4} \rightarrow \mathbb{R}^{4}(n)$, otherwise parallel space would have no physical meaning. A quantity $v(n)=n v(1)$, obtained from a quantity of $\mathbb{R}^{4}$, is not transformed again when going back from $\mathbb{R}^{4}$ (n) to $\mathbb{R}^{4}$, which means that the velocity of the spacecraft is $v(1)$ when returning to $\mathbb{R}^{4}$. This is in contrast to a quantity like $\Delta t(n)$ that transforms into $\Delta \mathrm{T}$. The reason for this non-symmetric behavior is that $\Delta t(n)$ is a quantity from $\mathbb{R}^{4}(n)$ and thus is being transformed. The spacecraft is assumed to make a transition from $\mathbb{R}^{4}$ into $\mathbb{R}^{4}$ (n) at velocity $v$ (or ). Since energy needs to be conserved in $\mathbb{R}^{4}$, the kinetic energy of the spacecraft remains unchanged upon reentry.

The value of $n$ is obtained from the following formula, Eq. (12), relating the field strength of the gravitophoton field, $g^{+}{ }_{g p}$, with the gravitational field strength, $g_{g}$, produced by the spacecraft itself,

$$
n=\frac{g_{g p}^{+}}{g_{g}} \frac{G_{g p}}{G} .
$$

For the transition into parallel space, a material with higher atomic number is needed, here magnesium $\mathrm{Mg}$ with $\mathrm{Z}=12$ is considered, which follows from the conversion equation for gravitophotons and gravitons into quintessence particles (stated without proof). Assuming a value of $g_{\mathrm{g}}=G M / R^{2}=10^{-7} \mathrm{~m} / \mathrm{s}^{2}$ for a mass of $10^{5} \mathrm{~kg}$ and a radius of $10 \mathrm{~m}$, a value of $g_{\mathrm{g}}=2 \times 10^{-5} \mathrm{~m} / \mathrm{s}^{2}$ is needed according to Eq. (12) provided that $\mathrm{Mg}$ as a material is used, a value of (see Table 1) $I n=1.3 \times 10^{7}$ is needed. If hydrogen was used, a magnetic induction of some $61 \mathrm{~T}$ would be needed, which hardly can be reached with present day technology.

From the numbers provided, it is clear that gravitophoton field propulsion, is far superior compared to chemical propulsion, or any other currently conceived propulsion system. For instance, an acceleration of $1 \mathrm{~g}$ could be sustained during a lunar mission. For such a mission only the acceleration phase is needed. A launch from the surface of the earth is foreseen with a spacecraft of a mass of some $1.5 \times 10^{5} \mathrm{~kg}$. With a magnetic induction of some $30 \mathrm{~T}$, compare Table 1 , a rotational speed of the torus of $v^{T}=10^{3} \mathrm{~m} / \mathrm{s}$, and a torus mass of $2 \times 10^{3}$ $\mathrm{kg}$, an acceleration larger than $1 \mathrm{~g}$ is produced, and thus the first half of the distance, $\mathrm{d}_{\mathrm{M}}$, to the moon is covered in some 2 hours, which follows from $t=\sqrt{2 d_{M} / g}$, resulting in a total flight time of 4 hours. A Mars mission, under the same assumptions as a flight to the moon, would achieve a final velocity of $v=g t=1.49 \times 10^{6}$ $\mathrm{m} / \mathrm{s}$. The total flight time to Mars with acceleration and deceleration is 3.4 days. Entering parallel space, a transition is possible at a speed of some $3 \times 10^{4} \mathrm{~m} / \mathrm{s}$ that will be reached after approximately 1 hour at a constant acceleration of $1 \mathrm{~g}$. In parallel space the velocity increases to $0.4 \mathrm{c}$, reducing total flight time to some 2.5 hours [14].

\section{Technical Realization of Field Pro- pulsion System}

From the discussion in Section 3.1 it has become clear that a large magnetic field is needed to produce an appreciable Heim-Lorentz force. In addition, Eq. (9) shows that the velocity of the electrons in the current loop must be large. Pulsed magnets can reach very high magnetic field strengths up to $60 \mathrm{~T}$ (Sandia Laboratories), and initially it was thought that these magnets could be used to provide the magnetic field to generating the Heim-Lorentz force needed for field propulsion. Regarding the equation for the Heim-Lorentz force, however, a high velocity of the electrons in the coil is needed. It is not sure that using a pulsed magnetic field this can be achieved in an effective way, since during the pulse period electrons need to be quickly accelerated to the speed $v_{\mathrm{c}}$ of the Cooper pairs, see below. The effectiveness of a pulsed magnet system depends critically on the ratio of pulse time and acceleration time. Furthermore, it is not clear how a rapidly time-varying Heim-Lorentz force would act on the structure of the spacecraft. A more detailed analysis would have to be carried out. At present, the usage of steady magnetic fields is preferred.

\subsection{Heim Field Propulsion Device}

If we look at the electron speed in metals, it is found that electron velocity is proportional to the applied electric field E. Electrons collide with the ions of the lattice and the time $\tau$ between these collisions is available to accelerate the electron. A brief calculation shows that $\tau_{\mathrm{c}}$ $\approx 10^{-15} \mathrm{~s}$. For a field strength of $\mathrm{E}=10 \mathrm{~V} / \mathrm{m}$ one obtains a speed of some $1 \mathrm{~cm} / \mathrm{s}$ for the electron. Hence, no metallic conductor in a non-superconducting state can be used.

At critical temperature $\mathrm{T}_{\mathrm{c}}$ some materials become superconductors that is, their resistance goes to 0. Superconductors have an energy gap of some $\mathrm{E}_{\mathrm{g}} \approx 3.5 \mathrm{kT}_{\mathrm{c}}$. This energy gap separates superconducting electrons below from normal electrons above the gap. At temperatures below $T_{c}$, electrons are coupled in pairs, called Cooper pairs, which are bosons. The exact formation of Cooper pairs is not known. The coupling of the electron pairs seems to be via phonons, generated by electron movement through the lattice of the superconductor. The size of a Cooper pair is some $10^{3} \mathrm{~nm}$. The crystal lattice contains defects that lead to an energy transfer $\Delta \mathrm{E}$ from the electron gas to the lattice. $\Delta \mathrm{E}$ must be smaller than $\mathrm{E}_{\mathrm{g}}$ otherwise the Cooper pairs are destroyed. 
The speed of the Cooper pairs can be calculated in a coordinate system where the electron gas is at rest and the lattice is moving, applying classical energy and momentum conservation. Decelerating the grid means that Cooper pairs gain energy. The maximum amount of energy that a Cooper pair can absorb is $\mathrm{E}_{\mathrm{g}}$, otherwise it is lifted in the band above, and superconductivity is lost. Therefore the simple ansatz

$$
\frac{1}{2} m v_{c}^{2}=E_{g}=3.5 k T_{c}
$$

can be used, $v_{\mathrm{c}}$ denoting the velocity of a Cooper pair. At temperature $\mathrm{T}_{\mathrm{c}}=10 \mathrm{~K}$ a speed of $v_{\mathrm{c}}=10^{4} \mathrm{~m} / \mathrm{s}$ is obtained.

\subsection{Magnetic Field Generation}

In order to obtain a viable space propulsion system the Heim-Lorentz formula requires magnetic fields of several tens of Tesla and current densities of several hundred $\mathrm{A} / \mathrm{mm}^{2}$. It is an experimental fact that high density magnetic fields destroy superconductivity. Type I (metals) superconductors have small $\mathrm{B}_{\mathrm{C}}$. Type II (alloys) superconductors have higher $\mathrm{B}_{\mathrm{C}}$. The picture, Fig. 7, plotted from data taken from the internet, shows the critical current density, $\boldsymbol{j}=2 e n \boldsymbol{v}$, at $4.2 \mathrm{~K}(\mathrm{He})$. At $25 \mathrm{~T}$ a current density of some $448 \mathrm{~A} / \mathrm{mm}^{2}$ can be sustained, which comes close to the value of $600 \mathrm{~A} / \mathrm{mm}^{2}$ assumed for field propulsion. Values of $60 \mathrm{~T}$, needed to transit into parallel space, so far have not been reached.

In the following, a brief discussion on the state of the art of producing high magnetic fields is given. For the fusion reactor Iter to be built in France in the next decade, a magnetic field strength of $9.7 \mathrm{~T}$ at $4.5 \mathrm{~K}$ carrying a current of $80 \mathrm{kA}$ using a $316 \mathrm{LN}$ stainless steel jacket with a diameter $40.7 \mathrm{~mm}$ was reached. This magnetic field is, however, not large enough for field propulsion.

There is a new high temperature superconducting material available, $\mathrm{Nb} 3 \mathrm{Sn}$, that has reached $12 \mathrm{~T}$ with 3,000 $\mathrm{A} / \mathrm{mm}^{2}$ at $4.2 \mathrm{~K}$. Other suitable compounds are Bi2212, Bi-2223, and Y-123.

High field magnets need a careful mechanical design because the Lorentz forces scale with $\mathrm{B}^{2}$. Nb3Sn can withstand up to $150 \mathrm{MPa}$ compressive force. In principle, a large current is better since lower number of turns is needed and therefore lower self-inductance.

This also means better superconductor volume efficiency or lower stored energy and thus lower peak temperature. However, the brittle $\mathrm{Nb}_{3} \mathrm{Sn}$ superconductor material is difficult to be made into long wires.

\section{Conclusions}

In $G R$ the geometrization of spacetime gives rise to gravitation. In Heim's theory four additional internal coordinates are introduced that affect events in our spacetime. Four subspaces can be discerned in this $8 \mathrm{D}$ world. From these four subspaces 12 partial met- ric tensors, termed hermetry forms, can be constructed that have direct physical meaning.

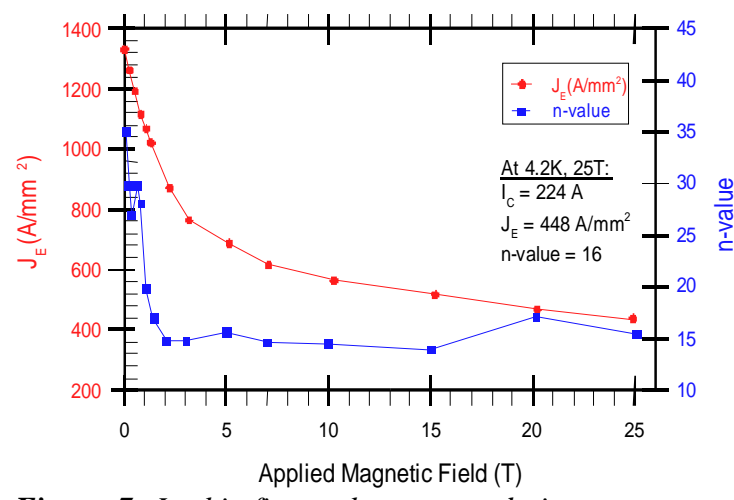

Figure 7: In this figure the current desity versus magnetic field at a temperature of $4.2 \mathrm{~K}(\mathrm{LHe})$ is plotted. At $25 \mathrm{~T}$ a current density of $448 \mathrm{~A} / \mathrm{mm}^{2}$ can be supported, a value that comes close to the $600 \mathrm{~A} / \mathrm{mm}^{2}$ needed for field propulsion.

In this way Einstein's geometrization principle was extended to construct a poly-metric that describes all known physical interactions, and also predicts two additional like gravitational forces that may be both attractive and repulsive. The theory predicts the conversion of photons into gravitophotons, denoted as the fifth fundamental interaction. The sixth fundamental interaction allows the conversion of gravitophotons and gravitons (spacecraft) into the repulsive vacuum or quintessence particles. Because of their repulsive character, the gravitational potential of the spacecraft is being reduced, requiring either a reduction of the gravitational constant or a speed of light larger than the vacuum speed of light. Both possibilities must be ruled out if the predictions of $L Q T$ and Heim theory are accepted, concerning the existence of a minimal surface. That is, spacetime is a quantized (discrete) field and not continuous. A lower value of $G$ or a higher value of $c$ clearly violate the concept of minimal surface. Therefore, in order to resolve this contradiction, the existence of a parallel space is postulated in which covariant laws of physics hold, but fundamental constants are different, see Eq. (11). The conditions for a transition in such a parallel space are given in Eq. (12).

It is most interesting to see that the consequent geometrization of physics, as suggested by Einstein in 1950 [9] starting from $G R$ and incorporating quantum theory along with the concept of spacetime as a quantized field as used by Heim and recently in $L Q T$, leads to major changes in fundamental physics and would allow to construct a completely different space propulsion system.

The technology for magnetic field generation seems to be sufficient to measure the Heim-Lorentz force, since a magnetic field of $18 \mathrm{~T}$ is feasible, but it must be provided over a diameter of some $3 \mathrm{~m}$, according to Table 1 to measuring a change in acceleration of $2 \times 10^{-5}$.

The goals of advanced propulsion as laid down by NASA in [15] are still valid and can only be obtained by novel physics. 


\section{Future Work}

Needless to say that Heim theory and also $L Q T$ harbor many unresolved issues. Heim theory needs to be put on a more rigorous mathematical basis. For instance, it is well known that Einstein's equations of general relativity can be derived from the variation of the EinsteinHilbert action. In the same way it needs to be shown how the hermetry forms can be used to derive all other known fundamental interactions. In particular, it should be clarified how the two additional gravitational interactions can be derived from a modified Einstein-Hilbert action. The conversion equation from photons into gravitophotons as well as the conversion of gravitophotons and gravitons into quintessence particles needs to be mathematically proved. So far, the authors have not derived the so called mass formula that is an eigenvalue equation leading to the mass spectrum of elementary particles as given by Heim.

With regard to the high currents needed, an alternative could be to use a hot plasma. Since currents of some $5 \times 10^{7} \mathrm{~A}$ are needed, this seems to be out of reach at present.

\section{Acknowledgment}

The authors are most grateful to Prof. P. Dr. Dr. A. Resch, director of IGW at Innsbruck University, for his continuous support in writing this paper. The second author was partly funded by Arbeitsgruppe Innovative Projekte (AGIP) and by Efre (EU)at the Ministry of Science and Education, Hanover, Germany.

Special thanks for help go to the friendly staff at the Loews Ventana Canyon Resort, Tucson, AZ where part of this paper was written.

\section{REFERENCES}

1. Zwiebach, R., Introduction to String Theory, Cambridge Univ. Press, 2004.

2. Liddle, A., An Introduction to Modern Cosmology, Wiley, 2003.

3. Lawrie, I.D.: A Unified Grand Tour of Theoretical Physics, 2nd ed., IoP 2002.

4. Smolin, L., Atoms of Space and Time, Scientific American, January 2004.

5. Rovelli, C., Quantum Gravity, 455 pp., Cambridge Univ. Press, 2004.

6. Strogatz, S., SYNC, Hyperion Books, New York, 2003.

7. Rovelli, C., Loop Quantum Gravity, Physics World, November 2003.

8. Zee, A., Quantum Field Theory in a Nutshell, Princeton Univ. Press, Princeton, 2003.

9. Einstein, On the Generalized Theory of Gravitation, Scientific American, April 1950, Vol 182, NO.4.
10. Ashtekar, A., et al., Background Independent Quantum Gravity:A Status Report, 125 pp., arXiv:GRqc/0404018 v1, 5 April 2004.

11. Heim, B., Vorschlag eines Weges einer einheitlichen Beschreibung der Elementarteilchen, Z. für Naturforschung, 32a, 1977, pp. 233-243.

12. Penrose, R., The Road to Reality, Chaps. 30-32, Vintage, 2004.

13. Dröscher,W., J. Hauser, AIAA 2004-3700, 40 AIAA/ASME/SAE/ASE, Joint Propulsion Conference \& Exhibit, Ft. Lauderdale, FL, 7-10 July, 2004, 21pp., see $\underline{w w w . u i b k . a c . a t / c / c b / c b 26}$.

14. Dröscher, W., J. Hauser, Heim Quantum Theory for Space Propulsion Physics, 2nd Symposium on New Frontiers and Future Concepts, STAIF, American Institute of Physics, CP 746, Ed. M.S. El-Genk 0-73540230-2/05, pp. 1430-1441.

15. Millis, M. NASA Breakthrough Propulsion Physics, Workshop Proceedings, NASA/CP-2004.

16. Veltman, M., Facts and Mysteries in Elementary Particle Physics, World Scientific, 2003.

17. Carrol, S. M., Spacetime and Geometry, AddisonWesley, San Francisco, 2004. 


\begin{tabular}{lccc}
\hline \multicolumn{1}{c}{ Generated by } & Messenger particles & Force & Coupling constant \\
\hline $\begin{array}{l}\text { real particles } \\
\text { virtual parti- } \\
\text { cles }\end{array}$ & graviton & attractive & $G_{g}$ \\
$\begin{array}{l}\text { Planck mass } \\
\text { vacuum }\end{array}$ & $\begin{array}{c}\text { quintessence or vac- } \\
\text { uum particle }\end{array}$ & $\begin{array}{c}\text { repulsive and attrac- } \\
\text { tive }\end{array}$ & $G_{g p}^{+}, G_{g p}^{-}=1 / 67^{2}$ \\
\hline
\end{tabular}

TABLE 2. The three gravitational interactions are related to different types of matter. The coupling constants were calculated using an idea by Heim.

TABLE 3. Classification scheme for physical interactions and messenger particles obtained from poly-metric in Heim space $\mathrm{H}^{8}$. Further explanation is given in the paper. Superscripts on subspaces indicate dimension. Subspaces $\mathrm{S}^{2}$ and $\mathrm{I}^{2}$ stand for organization and information, respectively. A hermetry form characterizes either a physical interaction or a messenger particle, and is associated with an admissible subspace. Either $\mathrm{S}^{2}$ or $\mathrm{I}^{2}$ need to be present in such a subspace in order to realize a physical event in our spacetime. Spaces $\mathrm{R}^{3}, \mathrm{~T}^{1}, \mathrm{~S}^{2}$ and $\mathrm{I}^{2}$ form the basis of Heim space $\mathrm{H}^{8}$. The additional four dimensions of $\mathrm{H}^{12}$ are not needed for describing physical interactions, but seem to steer probability amplitudes.

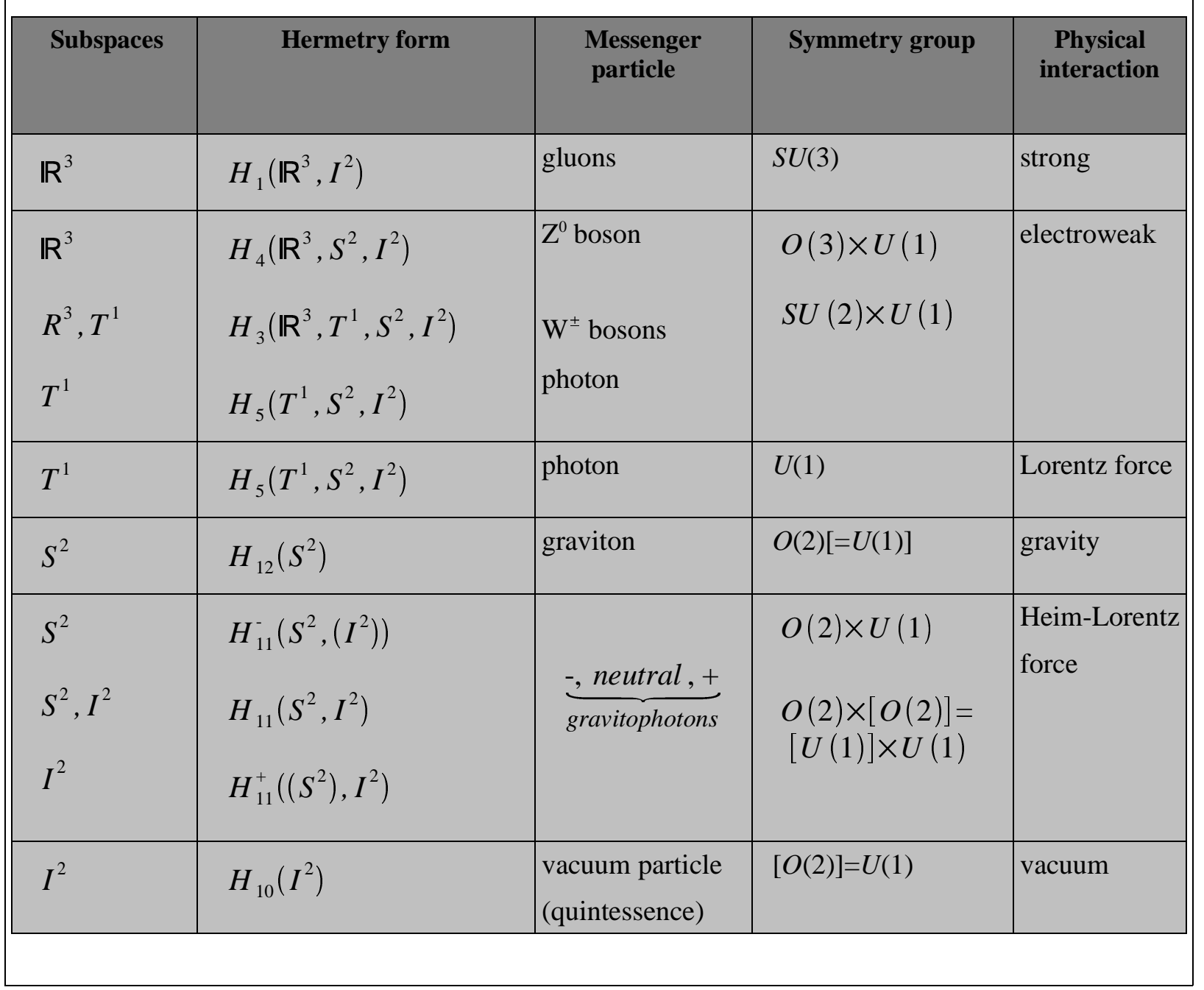

